\title{
The histone chaperone Asf1 is dispensable for direct de novo histone deposition in Xenopus egg extracts
}

\author{
Dominique Ray-Gallet • Jean-Pierre Quivy • \\ Herman W. W. Silljé • Erich A. Nigg • \\ Geneviève Almouzni
}

Received: 5 April 2007 /Revised: 16 May 2007 / Accepted: 20 May 2007

(C) Springer-Verlag 2007

\begin{abstract}
Histone chaperones that escort histones during their overall lifetime from synthesis to sites of usage can participate in various tasks. Their requirement culminates in the dynamic processes of nucleosome assembly and disassembly. In this context, it is important to define the exact role of the histone chaperone Asf1. In mammals, Asf1 interacts with two other chaperones, CAF-1 and HIRA, which are critical in DNA synthesis-coupled and synthesisuncoupled nucleosome assembly pathways, respectively. A key issue is whether Asfl is able or not to deposit histones onto DNA by itself in both pathways. Here, to delineate the precise role of Asfl in chromatin assembly, we used Xenopus egg extracts as a powerful system to assay de novo chromatin assembly pathways in vitro. Following characterization of both Xenopus Asf1 and p60 (CAF-1), we used immunodepletion strategies targeting Asfl, HIRA, or CAF-1. Strikingly, the depletion of Asfl led to the simultaneous depletion of HIRA and consequently impaired the DNA synthesis-independent nucleosome assembly pathway. The
\end{abstract}

Communicated by S. Henikoff

D. Ray-Gallet ·J.-P. Quivy • G. Almouzni $(\bowtie)$

Section de Recherche, UMR 218 du CNRS, Institut Curie,

26 rue d'Ulm,

75248 Paris Cedex 05, France

e-mail: almouzni@curie.fr

H. W. W. Silljé • E. A. Nigg

Department of Cell Biology,

Max-Planck Institute for Biochemistry,

Am Klopferspitz 18,

82152 Martinsried, Germany

Present address:

H. W. W. Silljé

Kiadis B.V.,

Zernikepark 6-8, 9747 AN,

Groningen, The Netherlands rescue of nucleosome assembly capacity in such extracts was effective when adding HIRA along with $\mathrm{H} 3 / \mathrm{H} 4$ histones, yet addition of Asf1 along with $\mathrm{H} 3 / \mathrm{H} 4$ histones did not work. Moreover, nucleosome assembly coupled to DNA repair was not affected in these Asf1/HIRA-depleted extracts, a pathway impaired by CAF-1 depletion. Thus, these data show that Asfl is not directly involved in de novo histone deposition during DNA synthesis-independent and synthesis-dependent pathways in egg extracts. Based on our results, it becomes important to consider the implications for Asf1 function during early development in Xenopus.

\section{Introduction}

Histone chaperones can play a variety of roles involved in histone metabolism (Loyola and Almouzni 2004). By definition, a histone chaperone is a factor that associates with histones to facilitate and control their interaction with other molecules without being a component of the final reaction product (Polo and Almouzni 2006). Their requirement culminates in the dynamic processes of nucleosome assembly and disassembly. Our current understanding of the assembly mechanisms enables to define two distinct pathways: (1) associated with DNA synthesis during DNA replication and specific repair events or (2) independently of DNA synthesis. The latter situation can be encountered as part of a histone exchange mechanism to introduce histone variants (Henikoff and Ahmad 2005; Kamakaka and Biggins 2005; Sarma and Reinberg 2005) or following eviction of histones from chromatin as proposed for activation of the PHO5 promoter in yeast (Boeger et al. 2003; Reinke and Horz 2003). To date, two H3/H4 histone chaperones, CAF-1 (chromatin assembly factor 1) and HIRA (histone regulating protein $\mathrm{A}$ ), have been characterized as directly involved 
in histone deposition per se. CAF-1, which consists of three subunits, p150, p60, and p48 in human cells (Ridgway and Almouzni 2000) is considered as the main histone chaperone that assists nucleosome assembly coupled to DNA synthesis (Groth et al. 2007) both during DNA replication (Smith and Stillman 1989) and nucleotide excision repair (NER) (Gaillard et al. 1996; Polo et al. 2006). In contrast, HIRA has been involved in a nucleosome assembly pathway independent of DNA synthesis in Xenopus egg extracts (Ray-Gallet et al. 2002) and during sperm nucleus decondensation in Drosophila (Loppin et al. 2005) and in mouse (van der Heijden et al. 2005). The isolation of a specific predeposition complex containing the replicative histone variant H3.1 together with CAF-1, distinct of another predeposition complex containing the replacement histone variant H3.3 together with HIRA supports this view (Tagami et al. 2004). The H3.3 variant, incorporated into chromatin independently of DNA synthesis (Ahmad and Henikoff 2002; Tagami et al. 2004) and proposed to mark transcriptionally active genes (Schwartz and Ahmad 2005), could thus use this latter chaperone (Loppin et al. 2005; Nakayama et al. 2007; van der Heijden et al. 2007).

Asf1 (Anti-silencing function 1), initially identified in $S$. cerevisiae (Le et al. 1997), is another H3/H4 chaperone whose orthologs in human consist of two genes, Asfla and Asflb, whereas only one Asfl was characterized in Drosophila. The copurification of Asfla and $\mathrm{b}$ with both CAF-1 in the H3.1 complex and HIRA in the H3.3 complex suggests that Asfla and b could potentially participate in both nucleosome assembly pathways coupled and uncoupled to DNA synthesis (Tagami et al. 2004). Furthermore, Asfl interacts directly with CAF-1p60 subunit and synergizes with CAF-1 to facilitate nucleosome assembly in Drosophila embryo extracts onto newly replicated DNA (Tyler et al. 1999) and in human cell extracts onto repaired DNA (Mello et al. 2002) or newly replicated DNA (Groth et al. 2005). Asfla, but not Asflb, interacts specifically with HIRA in human cells (Daganzo et al. 2003; Tagami et al. 2004), and both Asfla and HIRA have been involved in the formation of macroH2A-containing senescence-associated heterochromatic foci (SAHF; Zhang et al. 2005). The fact that both HIRA and p60 CAF-1 use similar motifs for binding to Asfla suggests a competing interaction with this chaperone (Tang et al. 2006). How this specificity applies to chromatin assembly is still an enigma. In any case, Asfl function seems to generally relate to disassembling and reassembling histones, as proposed based on work in S. cerevisiae in which Asfl importance was evaluated in the context of transcriptional activation (Adkins et al. 2004; Adkins and Tyler 2004; Schwabish and Struhl 2006).

Although the general view involving the histone chaperone Asfl in chromatin assembly is consistent with series of experiments in various organisms (Mousson et al. 2007), its exact position in the assembly line leading to nucleosome formation remains unclear. A major question is whether Asf1 is able or not to deposit histones onto DNA by itself or if it does act through another partner. In which case, its role would rather consist in being a "histone provider" as already proposed (Groth et al. 2005; Loyola and Almouzni 2004; Mello et al. 2002). Here, we aim to delineate the precise role of Asfl in chromatin assembly. For this, we use Xenopus egg extracts as a powerful source of histones and chaperones to test de novo chromatin assembly pathways in vitro (Almouzni and Mechali 1988; Laskey et al. 1977; RayGallet and Almouzni 2004). First, it was necessary to develop the adequate tools in Xenopus. We thus characterized both Xenopus Asf1 and p60 (CAF-1). Then, we could exploit immunodepletion strategies targeting Asf1, HIRA, p150, or p60 in Xenopus high-speed egg extracts (HSE) to examine the role of Asfl in histone deposition in comparison with the other chaperones. We carried out this analysis for both DNA synthesis-independent and synthesis-dependent nucleosome assembly pathways. Taken together our results argue that Asfl is not critically required for de novo histone deposition per se neither coupled nor uncoupled to DNA synthesis in Xenopus egg extracts.

\section{Materials and methods}

\section{Cloning and sequence analysis of Asf1 and p60} Xenopus cDNAs

Xenopus Asf1 cDNA isolated from a lambda gt11 Xenopus oocyte cDNA library (Clontech) was cloned in pBluescript SK (-) plasmid (accession number AY351903).

Xenopus p60 cDNA (clone XL061p11) was obtained from the NIBB Mochii normalized Xenopus tailbud library in the pBlueScript SK(-) plasmid (accession number AJ579309).

Fig. 1 Asf1 and p60 proteins in Xenopus Laevis. a Amino acid sequences of Xenopus Asfl (xAsf1), human Asfla (hAsfla), human Asflb (hAsf1b) and S. cerevisiae Asf1 (ScAsf1). The acidic amino acid stretch in ScAsfl is underlined. The two conserved aspartic residues D37 and D58, which are crucial for Asf1 complex formation with HIRA or CAF-1 p60, are indicated (Sanematsu et al. 2006; Tang et al. 2006). b Xenopus p60 (xp60), human $\mathrm{p} 60$ ( $h p 60)$ and $S$. cerevisiae p60 (Scp60). The sequence of the B domain of human/Xenopus HIRA, which is involved in Asfla binding is shown (HIRA residues that contact Asfla are in bold and underlined). HIRA B domain-like motifs of CAF-1 p60 are indicated by marking the residues that should contact Asfla (arrowhead for the two motifs in both Xenopus and human p60; star for the unique motif in yeast p60). The two arginine residues of the first HIRA B domain like-motif, R482 and R483, which are critical for HIRA and p60 CAF-1 binding, are indicated (Tang et al. 2006). We used ClustalW for alignments and shaded identical residues found at least in two of the sequences 


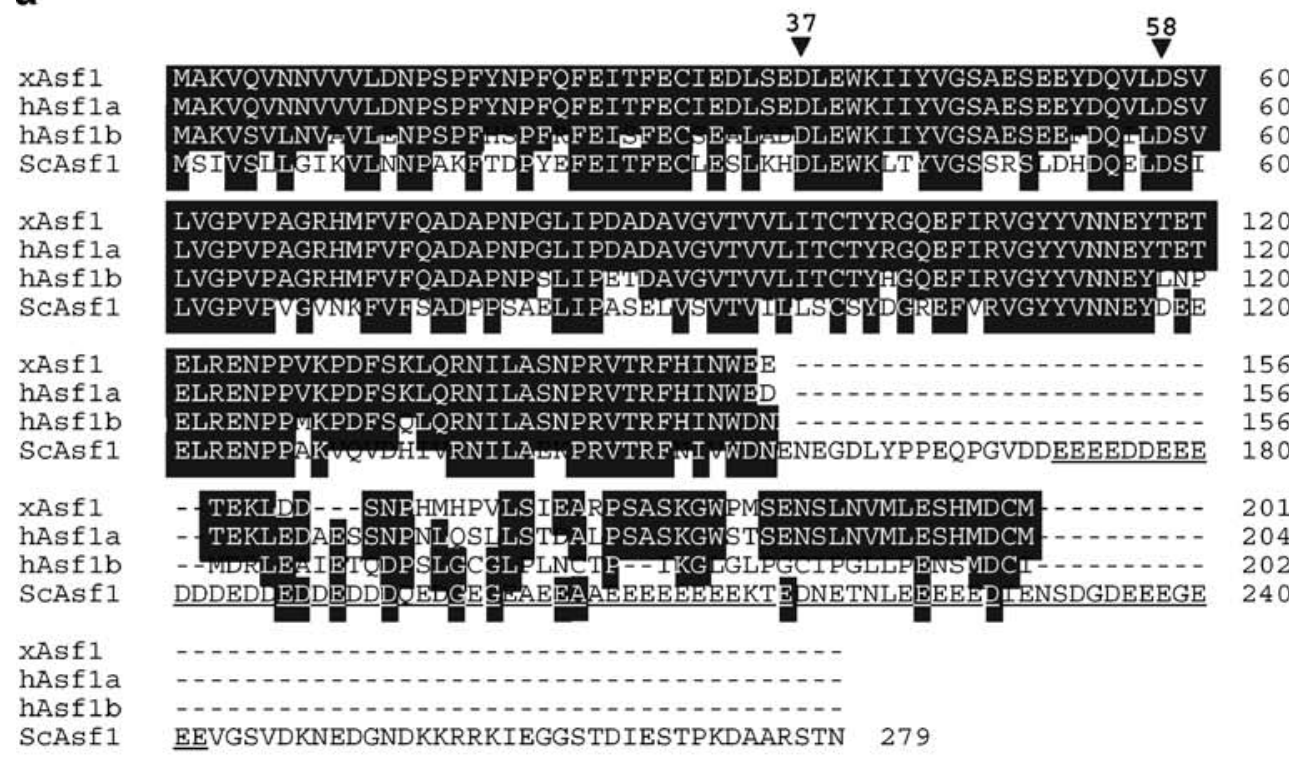

b

p60

WD repeats

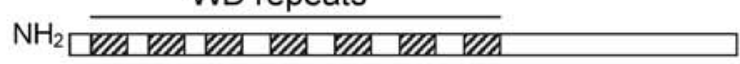

$\mathrm{COOH}$

HIRA B domain : 452- TRTADGRRRITPLCIAQLDTG -472

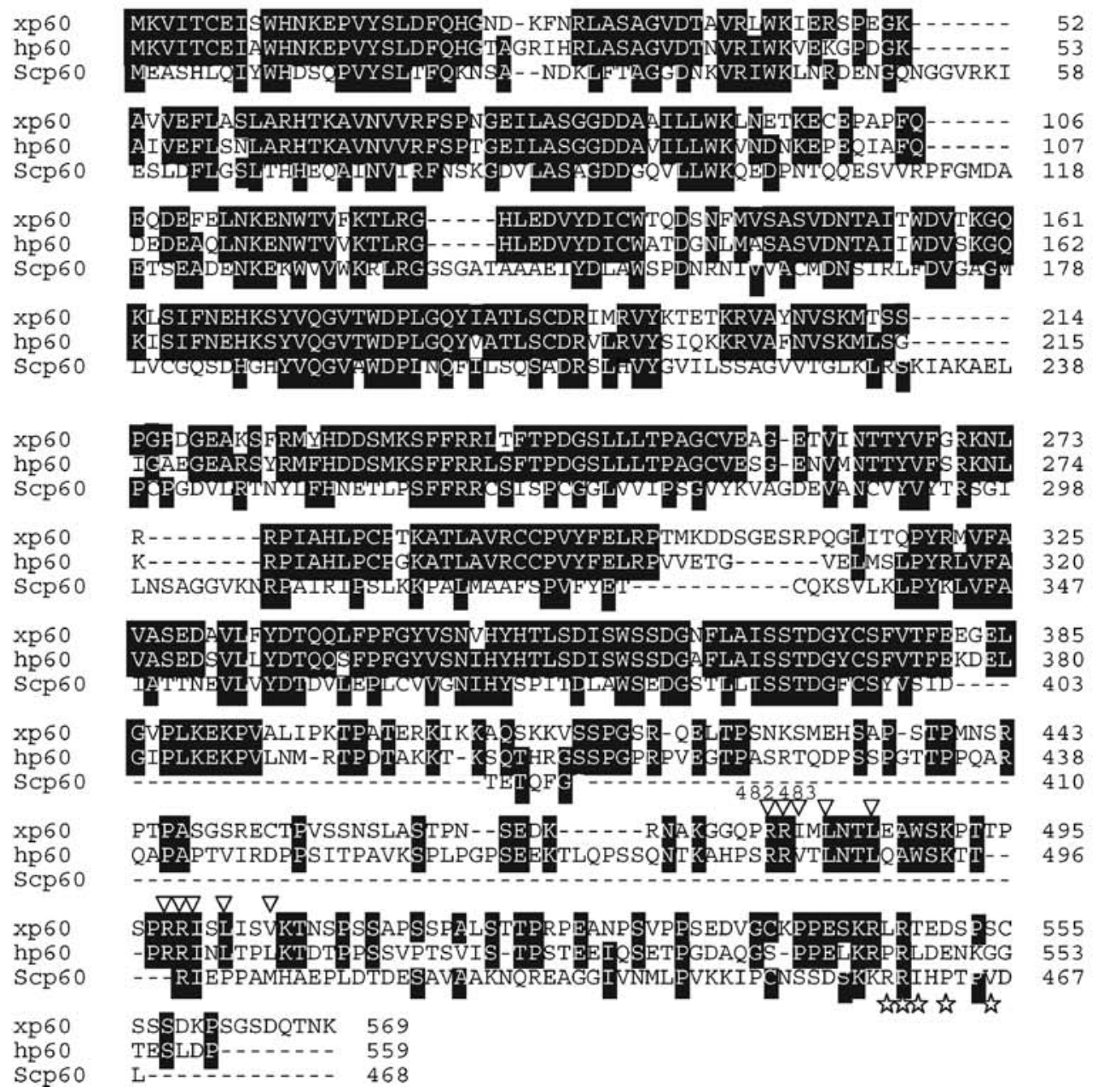


Asf1 and p60 recombinant Xenopus proteins and antibody production

PCR fragments containing the coding sequences of xAsf1 and xp60 cDNAs were subcloned in pGEX4T1 and PET 30 (for xAsf1) plasmids to produce GST and His fusion proteins, respectively. GST fusion and untagged HIRA Xenopus proteins were produced (Ray-Gallet et al. 2002). A rabbit polyclonal antiserum against Xenopus p60 was obtained by immunization with GST-p60 and against human Asfl proteins by immunization of rabbits with GST-hAsflb or GST-hAsfla (Groth et al. 2005).

Protein interactions and immunoprecipitations

A TNT-coupled rabbit reticulocyte lysate kit (Promega) was used for in vitro translation of the full-length HIRA and Asf1 Xenopus proteins from pBluescript clones and GST protein interactions performed (Ray-Gallet et al. 2002).

For HSE immunoprecipitation, $20 \mu \mathrm{l}$ of HSE were incubated with either anti-HIRA antiserum or preimmune serum for $2 \mathrm{~h}$ at $4^{\circ} \mathrm{C}$ in $10 \mathrm{mM}$ Hepes (pH 7.6), $150 \mathrm{mM}$ $\mathrm{KCl}, 0.5 \% \mathrm{NP} 40$ and protease inhibitors.

\section{Depletions and nucleosome assembly reactions}

The HSE, prepared as described (Almouzni 1998), were immunodepleted (Ray-Gallet et al. 2002) using antibodies against Xenopus p60, Xenopus HIRA (Ray-Gallet et al. 2002), Xenopus p150 (Quivy et al. 2001), and human Asflb (Groth et al. 2005) or mock-treated by incubation with noncoupled protein A sepharose.

In vitro nucleosome assembly reactions for $3 \mathrm{~h}$ at $23^{\circ} \mathrm{C}$ with HSE $(8 \mu \mathrm{l})$ used (1) nonirradiated pBS plasmid (-UV) for the pathway independent of DNA synthesis (2) or pBS plasmid irradiated with UV-C $\left(500 \mathrm{~J} / \mathrm{m}^{2} ;+\mathrm{UV}\right)$ in the presence of $\left(\alpha-{ }^{32} \mathrm{P}\right) \mathrm{dCTP}$ for the pathway coupled to nucleotide excision repair (NER) (Gaillard et al. 1999; Ray-Gallet and Almouzni 2004). Supercoiling and DNA synthesis analyzed by agarose gel electrophoresis were visualized by ethidium bromide staining (to detect Total DNA) and autoradiography (to detect labeled repaired DNA). For rescue of p150depleted HSE, human CAF-1 complex (p150, p60, p48) (15 ng) (Verreault et al. 1996) was added to the nucleosome assembly reaction. For complementation of Asf1/HIRA-depleted HSE, Xenopus His-Asf1 (10 ng) and/or untagged Xenopus HIRA protein (10 ng) and/or $(\mathrm{H} 3-\mathrm{H} 4)_{2}$ tetramers $(150 \mathrm{ng})$ purified from chicken erythrocytes (Simon and Felsenfeld 1979) were preincubated $10 \mathrm{~min}$ on ice before addition to the nucleosome assembly reaction after $10 \mathrm{~min}$ incubation.

\section{Results}

Xenopus Asf1 and p60 CAF-1

To dissect in the Xenopus system the position of Asfl in a chromatin assembly line and its relationship with CAF-1 and HIRA, we first cloned Asf1 and the p60 subunit of CAF-1 in Xenopus laevis. We found that Xenopus Asf1 cDNA encodes a protein of 201 amino acids which shares 94 and 70\% amino acid identity with human Asfla and Asflb, respectively (Sillje and Nigg 2001), and 45\% identity with the yeast S. cerevisiae Asfl (Le et al. 1997) (Fig. 1a). The C-terminal portion of these proteins is the least conserved and this divergence is highly pronounced when comparison is made with the yeast sequence which possesses an acidic stretch not observed in human and Xenopus proteins. The two aspartic residues in Asf1, Asp37, and Asp58, that were described as crucial for human Asfla complex formation with HIRA or CAF-1 p60 are conserved in the Xenopus protein (Sanematsu et al. 2006; Tang et al. 2006). Moreover, residues in Asf1 which are critical for $\mathrm{H} 3 / \mathrm{H} 4$ binding are also conserved in the Xenopus counterpart, as Val45, Asp54, Va194, and Tyr112 (English et al. 2006; Mousson et al. 2005; Natsume et al. 2007). The conservation of these critical amino acids emphasizes that Asfl may share common function(s) in various species.

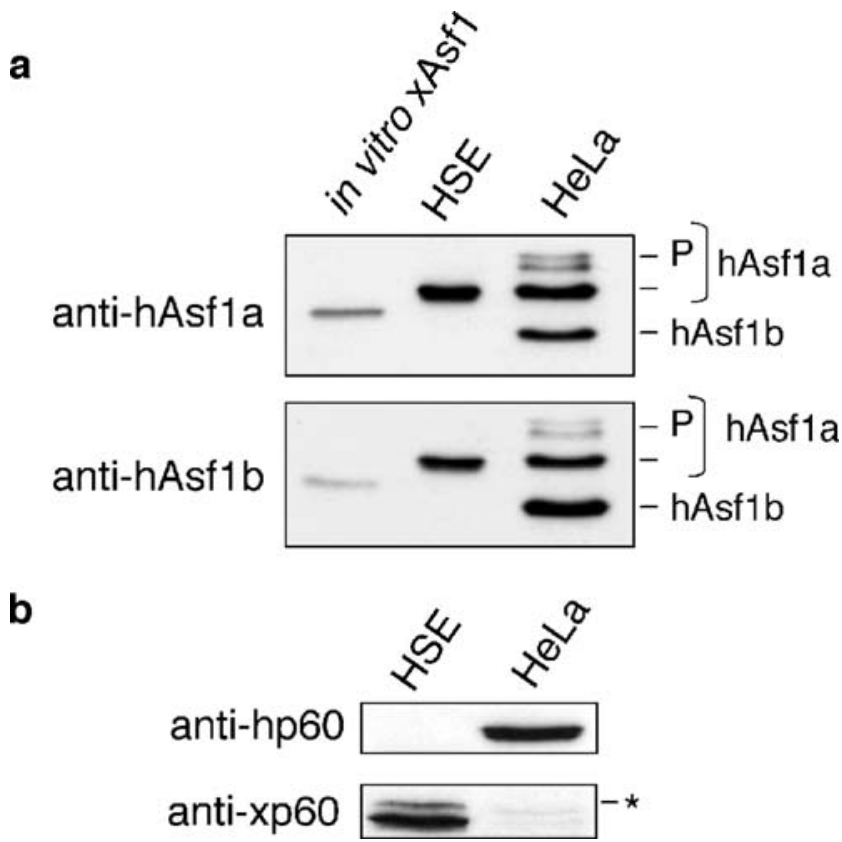

Fig. 2 Western blot analysis of Asf1 and p60 in Xenopus. a In vitro translated Xenopus Asfl, HSE $(1 \mu \mathrm{l})$ and human HeLa nuclear cell extracts $(20 \mu \mathrm{g})$ were analyzed by Western blotting with anti-human Asfla or anti-human Asflb antibodies. b HSE $(1 \mu l)$ and HeLa cell nuclear extracts $(20 \mu \mathrm{g})$ were analyzed by Western blotting using antibodies against human p60 (anti-hp60) or Xenopus p60 (anti-xp60). The asterisk indicates a minor band detected with the anti-xp60 antibody, potentially representing a p60 phosphorylated form 

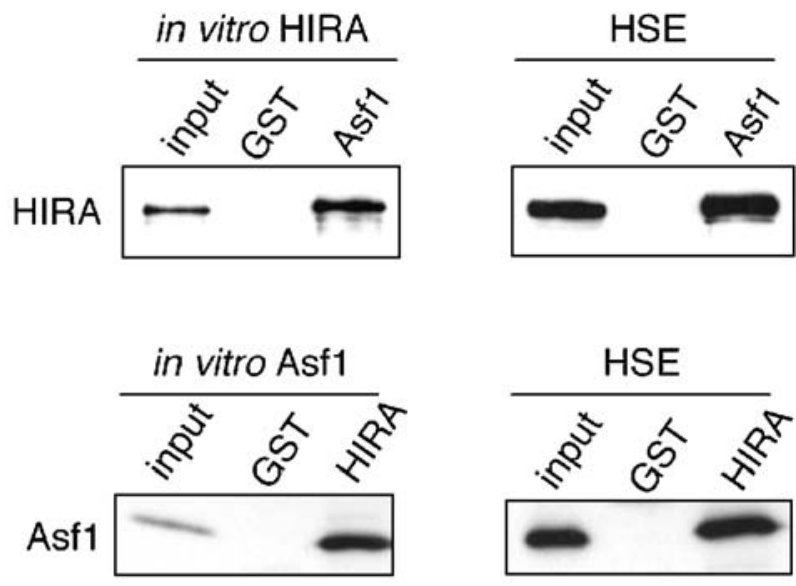

b
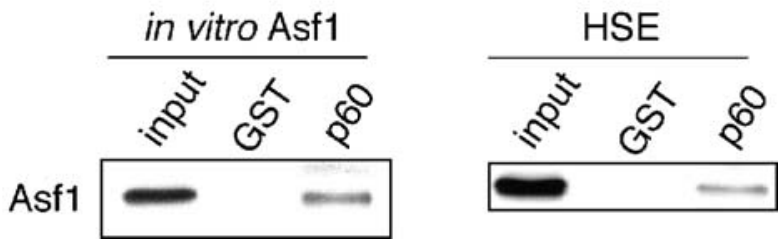

Fig. 3 Xenopus Asf1 interacts with HIRA and p60 Xenopus proteins in vitro. a In vitro translated Asfl or HSE were tested for binding to GST and GST-HIRA (HIRA) and conversely, in vitro translated HIRA or HSE for binding to GST or GST-Asf1 (Asfl). Input $(10 \%)$ and bound proteins analyzed by Western blotting using anti-Asf1 or antiHIRA antibodies. b In vitro translated Asfla or HSE were used for binding to GST or GST-p60 (p60) and bound proteins analyzed by Western blotting using anti-Asf1 antibody

Western blot analysis using antibodies raised against either human Asfla or Asflb, which recognize both proteins in human cell extracts, detected the in vitro translated Xenopus Asfl protein and one band in HSE (Fig. 2a). The Asf1 protein in egg extracts that exhibits a distinct migration profile when compared to in vitro translated protein can possibly be posttranslationally modified in Xenopus eggs. Indeed, the human counterparts can be phosphorylated by Tousled-like kinases (Tlk) (Sillje and Nigg 2001).

The Xenopus p60 cDNA encodes a protein of 569 amino acids sharing $63 \%$ amino acid identity with human p60 and $32 \%$ identity with yeast $S$. cerevisiae p60 (CAC2) (Fig. 1b). The most conserved region lies in the WD repeat domain in the N-terminal part of the protein. Interestingly, the two HIRA B domain-like motifs found in the C-terminal part of human CAF-1 p60 and whose the first one appears primarily responsible for Asfla binding (with the two critical arginine residues Arg482 and Arg483) are conserved in Xenopus (Tang et al. 2006). To search for the presence of p60 CAF-1 in Xenopus eggs, a specific rabbit polyclonal antibody was raised using a recombinant Xenopus p60 protein. Indeed, despite the conservation between human and Xenopus, an antibody raised against the human p60 was unable to reveal its Xenopus counter- part in egg extracts by Western blot analysis (Fig. 2b). We found significant amount of p60 in Xenopus eggs, which thus represent a valuable source of material to explore its function (Fig. 2b).

Xenopus Asf1 interacts with both HIRA and p60 in vitro

Xenopus GST-Asf1, GST-HIRA and GST-p60, in vitro translated HIRA and Asf1 proteins, and HSE were produced for pull-down assays. A specific interaction was detected between Asf1 and HIRA (Fig. 3a) and GST-p60 was able to pull down Asfl from an in vitro translation reaction or from HSE (Fig. 3b). These results demonstrate that the identified interactions of human Asfla with human HIRA (unpublished results; Daganzo et al. 2003; Tagami et al. 2004; Zhang et al. 2005) and human p60 (Mello et al. 2002) are conserved in Xenopus egg extracts. Thus, our

a

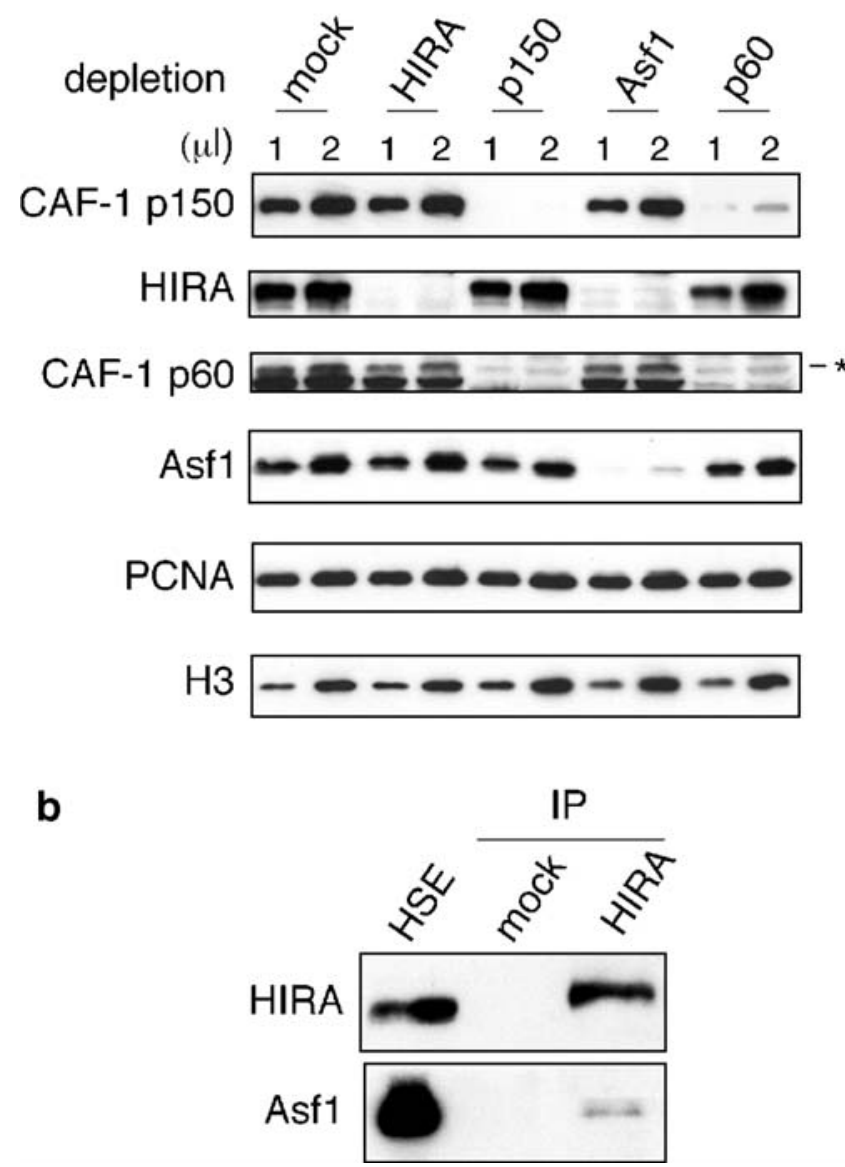

Fig. 4 Depletion of Asfl leads to co-depletion of HIRA in HSE. a 1 and $2 \mu \mathrm{l}$ of mock-, HIRA-, Asf1-, p60-, and p150-depleted HSE analyzed by Western blotting using the indicated antibodies. The asterisk is as in Fig. 2b a potential modified p60 form. b Input HSE $(10 \%)$ and immunoprecipitated proteins with either preimmune serum (mock) or HIRA antibody (HIRA) analyzed by Western blotting with anti-HIRA and anti-Asfl antibodies 
system, which recapitulates known properties of Asfl in somatic human cells, appears relevant to gain information concerning the role of Asf1 and its relationship with CAF-1 and HIRA in chromatin assembly.

Depletion of Asf1 from HSE leads to codepletion of HIRA

To study the role of Asf1 in chromatin assembly, HSE were immunodepleted for Asfl. We then tested the activity of the Asfl-depleted extracts in in vitro nucleosome assembly assays. For comparison, immunodepletions of HIRA, p60, and p150 CAF-1 from HSE were carried out in parallel using appropriate antibodies. These depletions were assessed by Western blot analysis as compared to a control HSE (mock-depleted; Fig. 4a). They were specific for the protein of interest and did not affect the amount of proliferating cell nuclear antigen (PCNA) nor histone $\mathrm{H} 3$ present in the extract. Although these chaperones are all known to interact with $\mathrm{H} 3$, the absence of codepletion of
$\mathrm{H} 3$ in the egg can be explained because the vast majority of the large maternal stores of histones $\mathrm{H} 3 / \mathrm{H} 4$ are sequestered by the chaperone N1/N2 (Kleinschmidt and Franke 1982; Laskey et al. 1978). Depletion of p60 resulted in a codepletion of p150 and conversely, showing that CAF-1 exists as a stable complex in these extracts. Strikingly, the Asf1 depletion led to an extensive codepletion of the HIRA protein, which compares to the efficiency achieved using antibodies directed against HIRA (Fig. 4a). In the latter case, the use of the HIRA polyclonal antiserum could not deplete Asfl (Fig. 4a and b), possibly due to the fact that Asfl is present in excess over HIRA or that binding of the antiHIRA antibody disrupts the association between HIRA and Asf1. In other species, Asfl proteins have been found interacting with several partners (Chimura et al. 2002; Emili et al. 2001; Moshkin et al. 2002), arguing that Asfl likely also exists independently of the HIRA complex. In any case, we can conclude that most of the HIRA proteins are associated with Asfl proteins as a stable complex in HSE.
Fig. 5 Depletion of Asf1/HIRA or CAF-1 and nucleosome assembly activity in HSE. a Nucleosome assembly reactions, with mock-, HIRA-, Asf1-, p60, or p150-depleted HSE, were performed using either nontreated plasmid $(-U V)$ to follow the pathway independent of DNA synthesis (left panel) or plasmid irradiated with $500 \mathrm{~J} / \mathrm{m}^{2}$ UV-C $(+U V)$ in presence of $\left(\alpha-{ }^{32} \mathrm{P}\right) \mathrm{dCTP}$ to examine the pathway coupled to DNA synthesis (right panel). After $3 \mathrm{~h}$ incubation, DNA was analyzed on agarose gel and visualized by ethidium bromide $(E t B r)$ or by autoradiography $\left({ }^{32} P\right)$. Migration positions of DNA plasmid form I (supercoiled), form II (nicked circular) and form Ir (closed circular) are indicated. b Graphs provide a quantification of the data ( with mock depletion as $100 \%$ reference)

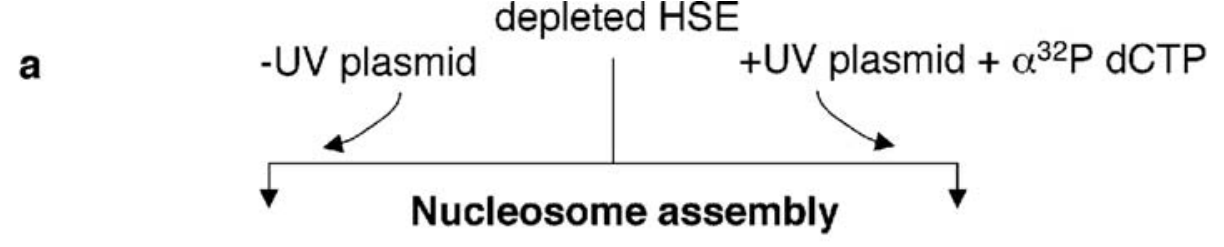

Independent of DNA synthesis

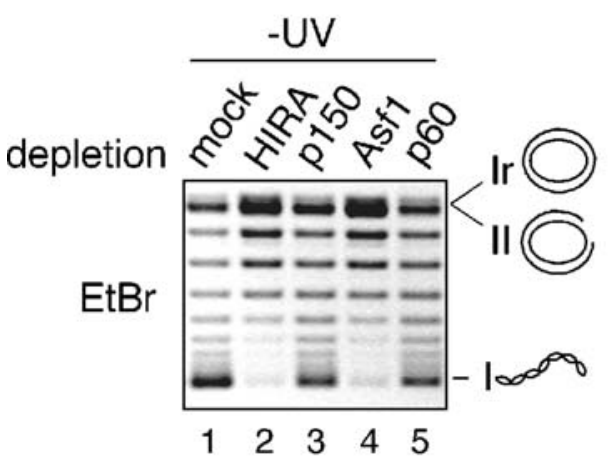

b

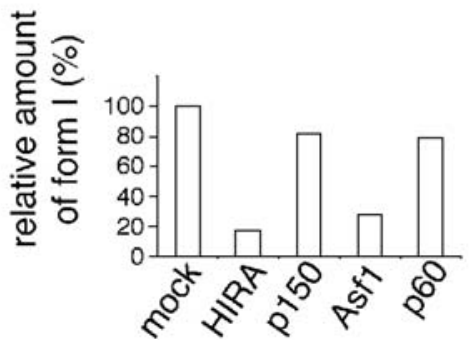

Associated with DNA synthesis

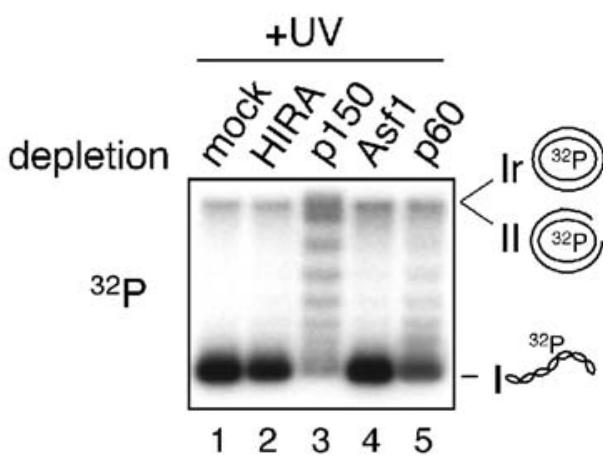

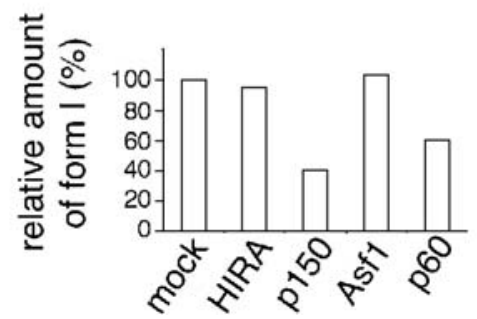


a

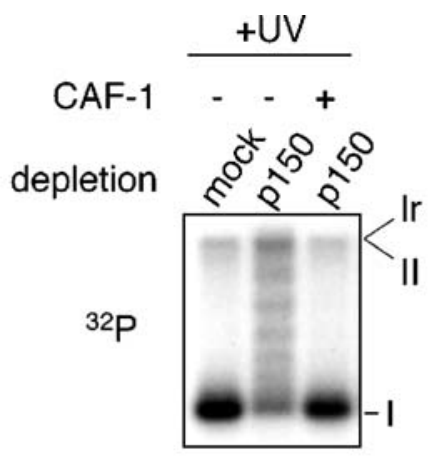

b

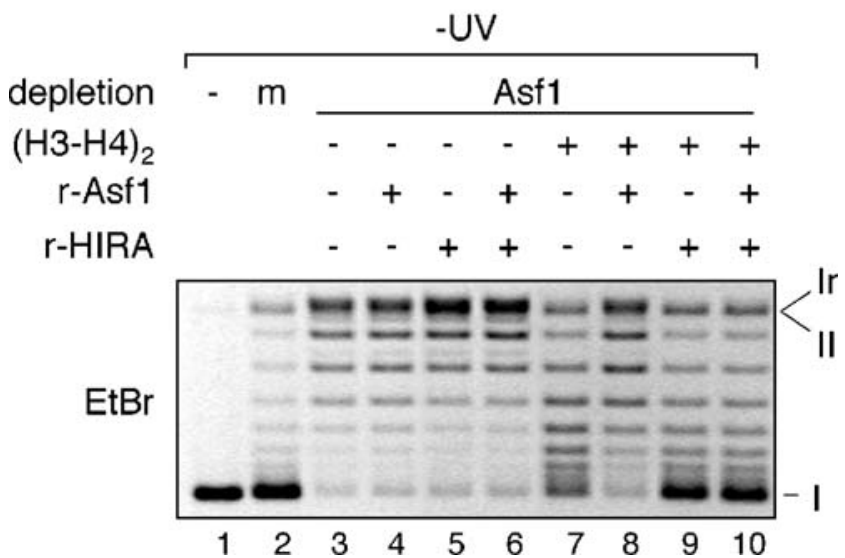

Fig. 6 Rescue of the nucleosome assembly capacities of p150- and Asf1/HIRA-depleted HSE. a Nucleosome assembly reactions using UV-treated plasmid $(+U V)$ and either mock- or p150-depleted HSE supplemented or not with human CAF-1 complex were analyzed as in Fig. 5a. b Various combinations of recombinant proteins ( $r$-HIRA and $r$-Asfl $)$ and $(\mathrm{H} 3-\mathrm{H} 4)_{2}$ tetramers were added to the nucleosome assembly reaction mix using nontreated plasmid $(-U V)$ and either mock $(m)$ or Asfl-depleted HSE and analyzed as in Fig. 5a. Input DNA run in parallel (lane 1). Migration positions are as in Fig. 5a

Asfl depletion, with HIRA codepletion, affects nucleosome assembly independent of DNA synthesis but not coupled to DNA repair

We compared the de novo nucleosome assembly capacities of HSE depleted for Asf1, HIRA, p60, and p150 in both DNA synthesis-coupled and synthesis-uncoupled pathways. To examine nucleosome assembly independent of DNA synthesis we used undamaged plasmid (-UV). In the HSE control (mock-depleted) or depleted for p60 or p150, nucleosome assembly occurred efficiently as revealed by the appearance of supercoiled plasmid (form I) after $3 \mathrm{~h}$ incubation (Fig. 5a, -UV: lanes 1, 3, and 5). In contrast, in HIRA- or Asf1-depleted extracts, this DNA synthesisindependent assembly was severely impaired (Fig. 5a, -UV: lanes 2 and 4). Next, to examine nucleosome assembly coupled to DNA synthesis, we performed a similar analysis using UV-irradiated plasmid (+UV). In this system, the repaired DNA is detected by the incorporation of radiolabeled nucleotides $\left(\alpha^{32} \mathrm{P}\right.$ dCTP). While the assembly pathway linked to DNA synthesis was intact in the control, HIRA- or Asf1-depleted HSE (Fig. 5a, +UV: lanes 1, 2, and 4 ), this assembly was affected in the p150- or p60-depleted HSE (Fig. 5a, +UV: lanes 3 and 5). Quantification of these data is shown on the graphs in Fig. 5b. Thus, in this system, Asfl appears dispensable for direct de novo histone deposition in the nucleosome assembly pathway coupled to DNA synthesis associated with NER whereas both p60 and p150 are essential. Remarkably, Asfl-depleted extracts codepleted for HIRA were impaired in their ability to assemble nucleosomes independently of DNA synthesis in a manner comparable to extracts depleted of HIRA alone.

Addition of Asf1 cannot rescue the nucleosome assembly capacity of Asf1/HIRA depleted extracts

Rescue experiments of the Asf1/HIRA-depleted extracts showed that neither the addition of Asfl or HIRA recombinant proteins nor $\mathrm{H} 3 / \mathrm{H} 4$ histones added separately is sufficient for recovery of DNA synthesis-independent nucleosome assembly (Fig. 6b, lanes 4, 6, and 8). In contrast, the simple addition of CAF-1 subunits is able to restore nucleosome assembly coupled to DNA repair in p150-depleted HSE (Fig. 6a). However, the combined addition of HIRA along with $\mathrm{H} 3 / \mathrm{H} 4$ markedly improved assembly efficiency of Asf1/HIRA-depleted extracts (Fig. 6b, lane 9) as seen for HIRA depleted extracts (RayGallet et al. 2002). Strikingly, a similar combined addition of Asfl along with $\mathrm{H} 3 / \mathrm{H} 4$ has no effect (Fig. 6b, lane 8), and addition of Asfl with both HIRA and $\mathrm{H} 3 / \mathrm{H} 4$ did not improve further the rescue (Fig. 6b compare lanes 9 and 10). Although we cannot totally exclude that the small amount of Asf1 remaining in the Asfl-depleted extracts (Fig. 4a) may contribute in combination with HIRA to the rescue of the nucleosome assembly, our results strongly suggest that Asfl is not critical for de novo deposition of histones per se in the assembly process independent of DNA synthesis using egg extracts.

\section{Discussion}

To assign to the histone chaperone Asfl a position in a chromatin assembly line and to relate it to CAF-1 and HIRA functions, we took advantage of the properties of Xenopus egg extracts. These extracts can be depleted of a chosen chaperone to evaluate its respective contribution to DNA synthesis-dependent or synthesis-independent de novo nucleosome assembly. To exploit the Xenopus system, it was necessary to first clone and identify Asf1 and p60 subunit of CAF-1 in Xenopus. Then, using recombinant proteins, we showed that Xenopus Asfl interacts with both p60 and HIRA (Fig. 3). Furthermore, the codepletion of HIRA from 
HSE using Asfl antibodies indicated that the HIRA protein is mostly complexed with Asfl in egg extracts (Fig. 4a). Although Asf1 and p60 interact in vitro, the depletion of either Asf1 or CAF-1 (p60 or p150) from HSE did not lead to their codepletion suggesting that only a small proportion of the proteins may exist in a stable complex in egg extracts (Fig. 4a). The Asf1/HIRA-depleted HSE remained efficient to assemble nucleosome coupled to DNA synthesis, a pathway impaired in CAF-1 depleted extracts (Fig. 5). In egg extracts, large amount of Asf1 appears therefore dispensable to assemble nucleosomes coupled to DNA repair. This is different from the situation in extracts derived from human somatic cells for which a similar depletion of Asf1 can lead to a severe defect in this nucleosome assembly pathway (Groth et al. 2005). Thus, the replicative pool of histones available for CAF-1 is not as limiting in egg extracts, possibly due to the existence of alternative storage forms, not necessarily mediated by Asf1. Indeed, in Xenopus eggs the maternal pool of $\mathrm{H} 3 / \mathrm{H} 4$ is associated with the chaperone N1/N2 (Kleinschmidt and Franke 1982; Laskey et al. 1978). In this context, we can therefore hypothesize that N1/N2 is the direct "histone provider" for CAF-1 and that Asf1 is dispensable for this function. In the Asf1/HIRAdepleted HSE, the nucleosome assembly independent of DNA synthesis was severely impaired to an extent comparable to the HIRA depletion (Fig. 5). This observation further supports the importance of HIRA in this pathway, as shown by the rescue obtained by a simple addition of HIRA and histones (Fig. 6b). Importantly, the readdition of Asf1 along with histones $\mathrm{H} 3 / \mathrm{H} 4$ failed to restore the assembly activity. Recent studies showed that Asfl interacts with H3H4 as dimers (Antczak et al. 2006; English et al. 2006; Natsume et al. 2007). Thus, we cannot exclude that the source of H3-H4 as tetramers that we used in our set up could account for a lack of efficiency in the Xenopus system. However, this seems unlikely because a similar set up was used successfully with human cell extracts (Groth et al. 2005; Mello et al. 2002), and recent data showed that Asf1 can disrupt (H3-H4) 2 tetramers to form Asf1-H3-H4 complex (Natsume et al. 2007). From our data, we emphasize that, although Asfl can promote reconstitution of nucleosomes when using pure components, a general property expected from any "histone chaperone" under such conditions (Green et al. 2005), we show here that this property does not suffice to make it a deposition factor.

Taken together, in the complex system of Xenopus egg extracts, the emerging picture is thus that Asf1 is either dispensable or likely functions as a "histone provider" for de novo histone deposition while CAF-1 and HIRA act as deposition factors in an assembly pathway. The specific properties of Xenopus egg extracts, which contain large amounts of the proteins needed for the rapid nuclear divisions occurring after fertilization in the absence of transcription, may explain why Asf1 appears not critically required at this stage. In egg extracts, CAF-1 and HIRA could already be present in complexes ready for histone deposition and in amounts not limiting for the in vitro nucleosome assembly assays (and therefore not depending on the Asf1 "histone provider" function). Alternatively, at this stage, they would function independently of Asfl with other "histone providers" such as N1/N2. Finally, we should bear in mind that the role of Asfl may also relate to nucleosome disassembly by disrupting $(\mathrm{H} 3-\mathrm{H} 4)_{2}$ tetramers. Yet, we could not evaluate this property, using naked DNA as a template for our in vitro de novo assembly assays. Indeed, the recent determination by several groups of the crystal structure of Asf1 bound to histones revealed that Asfl binds to a heterodimer of histones $\mathrm{H} 3 / \mathrm{H} 4$ (Antczak et al. 2006; English et al. 2006; Natsume et al. 2007). This is consistent with previous arguments, which put forward a similar CAF-1 and HIRA interaction with H3/H4 heterodimers (Tagami et al. 2004). The possible disruptive activity of Asf is consistent with its reported role in nucleosome disassembly in yeast (Adkins et al. 2004; Adkins and Tyler 2004). It will be important to further evaluate the role of Asfl in the context of chromatin templates and thus be able to test a combined function to coordinate both disassembly and reassembly events.

Finally, to properly determine the relative importance of Asf1 in comparison with CAF-1 and HIRA, it will be interesting to examine its requirement during development at specific stages. Indeed, a critical role for CAF-1 was demonstrated during the early cleavage stages of Xenopus embryogenesis (Quivy et al. 2001) and knock down of HIRA in mouse resulted in embryonic lethality at the gastrulation stage (Roberts et al. 2002). HIRA is involved in the incorporation of the histone variant H3.3 in the male pronucleus before the first round of DNA replication in Drosophila (Loppin et al. 2005) and mouse (van der Heijden et al. 2005), an event which appears critical for the embryo development. Determining how Asfl is required or not during these development steps should help to understand its specific function at the level of an organism at key developmental stages.

In conclusion, our results emphasize that Asf1 is not directly involved in de novo histone deposition in Xenopus egg extracts, a role played by CAF-1 and HIRA in DNA synthesis-coupled and synthesis-uncoupled pathways, respectively.

Acknowledgement We thank NIBB for providing us Xenopus p60 cDNA clone. We are grateful to F. Sangrado for Xenopus p60 antibodies. We thank A. Verreault for human CAF-1 complex. We also thank Adam Cook for critical reading of the manuscript. This work was supported by la Ligue Nationale contre le Cancer, the Commissariat à l'Energie Atomique, European contract RTN, NoE Epigenome, and Canceropole. HHWS and EAN acknowledge support from the Max-Planck-Society. 


\section{References}

Adkins MW, Tyler JK (2004) The histone chaperone Asflp mediates global chromatin disassembly in vivo. J Biol Chem 279:5206952074

Adkins MW, Howar SR, Tyler JK (2004) Chromatin disassembly mediated by the histone chaperone Asfl is essential for transcriptional activation of the yeast $\mathrm{PHO} 5$ and $\mathrm{PHO} 8$ genes. Mol Cell 14:657-666

Ahmad K, Henikoff S (2002) The histone variant H3.3 marks active chromatin by replication-independent nucleosome assembly. Mol Cell 9:1191-1200

Almouzni G (1998) Assembly of chromatin and nuclear structures in Xenopus egg extracts. In Chromatin, a practical approach. Oxford University Press, Oxford, pp 195-218

Almouzni G, Mechali M (1988) Assembly of spaced chromatin promoted by DNA synthesis in extracts from Xenopus eggs. EMBO J 7:665-672

Antczak AJ, Tsubota T, Kaufman PD, Berger JM (2006) Structure of the yeast histone H3-ASF1 interaction: implications for chaperone mechanism, species-specific interactions, and epigenetics. BMC Struct Biol 6:26

Boeger H, Griesenbeck J, Strattan JS, Kornberg RD (2003) Nucleosomes unfold completely at a transcriptionally active promoter. Mol Cell 11:1587-1598

Chimura T, Kuzuhara T, Horikoshi M (2002) Identification and characterization of CIA/ASF1 as an interactor of bromodomains associated with TFIID. Proc Natl Acad Sci USA 99:9334-9339

Daganzo SM, Erzberger JP, Lam WM, Skordalakes E, Zhang R, Franco AA, Brill SJ, Adams PD, Berger JM, Kaufman PD, Krawitz DC, Kama T (2003) Structure and function of the conserved core of histone deposition protein Asfl. Curr Biol 13:2148-2158

Emili A, Schieltz DM, Yates JR 3rd, Hartwell LH (2001) Dynamic interaction of DNA damage checkpoint protein Rad53 with chromatin assembly factor Asf1. Mol Cell 7:13-20

English CM, Adkins MW, Carson JJ, Churchill ME, Tyler JK (2006) Structural basis for the histone chaperone activity of Asf1. Cell 127:495-508

Gaillard PH, Martini EM, Kaufman PD, Stillman B, Moustacchi E, Almouzni G (1996) Chromatin assembly coupled to DNA repair: a new role for chromatin assembly factor I. Cell 86:887-896

Gaillard PH, Roche D, Almouzni G (1999) Nucleotide excision repair coupled to chromatin assembly. In: Methods Mol. Biol. Humana, Totowa, NJ pp 231-243

Green EM, Antczak AJ, Bailey AO, Franco AA, Wu KJ, Yates JR 3rd, Kaufman PD (2005) Replication-independent histone deposition by the HIR complex and Asf1. Curr Biol 15:2044-2049

Groth A, Ray-Gallet D, Quivy JP, Lukas J, Bartek J, Almouzni G (2005) Human Asfl regulates the flow of S phase histones during replicational stress. Mol Cell 17:301-311

Groth A, Rocha W, Verreault A, Almouzni G (2007) Chromatin challenges during DNA replication and repair. Cell 128:721-733

Henikoff S, Ahmad K (2005) Assembly of variant histones into chromatin. Annu Rev Cell Dev Biol 21:133-153

Kamakaka RT, Biggins S (2005) Histone variants: deviants? Genes Dev 19:295-310

Kleinschmidt JA, Franke WW (1982) Soluble acidic complexes containing histones $\mathrm{H} 3$ and $\mathrm{H} 4$ in nuclei of Xenopus laevis oocytes. Cell 29:799-809

Laskey RA, Mills AD, Morris NR (1977) Assembly of SV40 chromatin in a cell-free system from Xenopus eggs. Cell 10:237-243

Laskey RA, Honda BM, Mills AD, Finch JT (1978) Nucleosomes are assembled by an acidic protein which binds histones and transfers them to DNA. Nature 275:416-420
Le S, Davis C, Konopka JB, Sternglanz R (1997) Two new S-phasespecific genes from Saccharomyces cerevisiae. Yeast 13: $1029-1042$

Loppin B, Bonnefoy E, Anselme C, Laurencon A, Karr TL, Couble P (2005) The histone H3.3 chaperone HIRA is essential for chromatin assembly in the male pronucleus. Nature 437:13861390

Loyola A, Almouzni G (2004) Histone chaperones, a supporting role in the limelight. Biochim Biophys Acta 15:1-3

Mello JA, Sillje HH, Roche DM, Kirschner DB, Nigg EA, Almouzni G (2002) Human Asf1 and CAF-1 interact and synergize in a repaircoupled nucleosome assembly pathway. EMBO Rep 3:329-334

Moshkin YM, Armstrong JA, Maeda RK, Tamkun JW, Verrijzer P, Kennison JA, Karch F (2002) Histone chaperone ASF1 cooperates with the Brahma chromatin-remodelling machinery. Genes Dev 16:2621-2626

Mousson F, Lautrette A, Thuret JY, Agez M, Courbeyrette R, Amigues B, Becker E, Neumann JM, Guerois R, Mann C, Ochsenbein F (2005) Structural basis for the interaction of Asf1 with histone $\mathrm{H} 3$ and its functional implications. Proc Natl Acad Sci USA 102:5975-5980

Mousson F, Ochsenbein F, Mann C (2007) The histone chaperone Asfl at the crossroads of chromatin and DNA checkpoint pathways. Chromosoma 116:79-93

Nakayama T, Nishioka K, Dong YX, Shimojima T, Hirose S (2007) Drosophila GAGA factor directs histone H3.3 replacement that prevents the heterochromatin spreading. Genes Dev 21:552-561

Natsume R, Eitoku M, Akai Y, Sano N, Horikoshi M, Senda T (2007) Structure and function of the histone chaperone CIA/ASF1 complexed with histones H3 and H4. Nature 446:338-341

Polo SE, Almouzni G (2006) Chromatin assembly: a basic recipe with various flavours. Curr Opin Genet Dev 16:104-111

Polo SE, Roche D, Almouzni G (2006) New histone incorporation marks sites of UV repair in human cells. Cell 127:481-493

Quivy JP, Grandi P, Almouzni G (2001) Dimerization of the largest subunit of chromatin assembly factor 1 : importance in vitro and during Xenopus early development. EMBO J 20:2015-2027

Ray-Gallet D, Almouzni G (2004) DNA synthesis-dependent and independent chromatin assembly pathways in Xenopus egg extracts. Methods Enzymol 375:117-131

Ray-Gallet D, Quivy JP, Scamps C, Martini EM, Lipinski M, Almouzni G (2002) HIRA is critical for a nucleosome assembly pathway independent of DNA synthesis. Mol Cell 9:1091-1100

Reinke H, Horz W (2003) Histones are first hyperacetylated and then lose contact with the activated PHO5 promoter. Mol Cell 11:1599-1607

Ridgway P, Almouzni G (2000) CAF-1 and the inheritance of chromatin states: at the crossroads of DNA replication and repair. J Cell Sci 113:2647-2658

Roberts C, Sutherland HF, Farmer H, Kimber W, Halford S, Carey A, Brickman JM, Wynshaw-Boris A, Scambler PJ (2002) Targeted mutagenesis of the hira gene results in gastrulation defects and patterning abnormalities of mesoendodermal derivatives prior to early embryonic lethality. Mol Cell Biol 22:2318-2328

Sanematsu F, Takami Y, Barman HK, Fukagawa T, Ono T, Shibahara $\mathrm{K}$, Nakayama $\mathrm{T}$ (2006) Asfl is required for viability and chromatin assembly during DNA replication in vertebrate cells. J Biol Chem 281:13817-13827

Sarma K, Reinberg D (2005) Histone variants meet their match. Nat Rev Mol Cell Biol 6:139-149

Schwabish MA, Struhl K (2006) Asfl mediates histone eviction and deposition during elongation by RNA polymerase II. Mol Cell 22:415-422

Schwartz BE, Ahmad K (2005) Transcriptional activation triggers deposition and removal of the histone variant H3.3. Genes Dev $19: 804-814$ 
Sillje HH, Nigg EA (2001) Identification of human Asf1 chromatin assembly factors as substrates of Tousled-like kinases. Curr Biol 11:1068-1073

Simon RH, Felsenfeld G (1979) A new procedure for purifying histone pairs $\mathrm{H} 2 \mathrm{~A}+\mathrm{H} 2 \mathrm{~B}$ and $\mathrm{H} 3+\mathrm{H} 4$ from chromatin using hydroxylapatite. Nucleic Acids Res 6:689-696

Smith S, Stillman B (1989) Purification and characterization of CAFI, a human cell factor required for chromatin assembly during DNA replication in vitro. Cell 58:15-25

Tagami H, Ray-Gallet D, Almouzni G, Nakatani Y (2004) Histone H3.1 and H3.3 complexes mediate nucleosome assembly pathways dependent or independent of DNA synthesis. Cell 116:51-61

Tang Y, Poustovoitov MV, Zhao K, Garfinkel M, Canutescu A, Dunbrack R, Adams PD, Marmorstein R (2006) Structure of a human ASF1aHIRA complex and insights into specificity of histone chaperone complex assembly. Nat Struct Mol Biol 13:921-929

Tyler JK, Adams CR, Chen SR, Kobayashi R, Kamakaka RT, Kadonaga JT (1999) The RCAF complex mediates chromatin assembly during DNA replication and repair. Nature 402:555-560 van der Heijden GW, Dieker JW, Derijck AA, Muller S, Berden JH, Braat DD, van der Vlag J, de Boer P (2005) Asymmetry in Histone $\mathrm{H} 3$ variants and lysine methylation between paternal and maternal chromatin of the early mouse zygote. Mech Dev 122:1008-1022

van der Heijden GW, Derijck AA, Posfai E, Giele M, Pelczar P, Ramos L, Wansink DG, van der Vlag J, Peters AH, de Boer P (2007) Chromosome-wide nucleosome replacement and H3.3 incorporation during mammalian meiotic sex chromosome inactivation. Nat Genet 39:251-258

Verreault A, Kaufman PD, Kobayashi R, Stillman B (1996) Nucleosome assembly by a complex of CAF-1 and acetylated histones H3/H4. Cell 87:95-104

Zhang R, Poustovoitov MV, Ye X, Santos HA, Chen W, Daganzo SM, Erzberger JP, Serebriiskii IG, Canutescu AA, Dunbrack RL, Pehrson JR, Berger JM, Kaufman PD, Adams PD (2005) Formation of MacroH2A-containing senescence-associated heterochromatin foci and senescence driven by ASF1a and HIRA. Dev Cell 8:19-30 